\title{
Letter to the Editor and Global Public: Lockdown Block Efficiency Equivalent Devices for COVID-19 Pandemic
}

\author{
Yi Yu Lai \\ Innoen Gravitational Institute, L6W1V1, Ontario, Canada \\ yylai[at]innoen.org
}

To date, the only successful strategy for blocking COVID-19 is by a large-scaled lockdown at the population level. China, Thailand, and Vietnam have successfully taken this strategy to control their pandemic. The root cause is the RNA coronavirus evolution pattern, if the SARS-VOR-2 can continuously get fresh human bodies as relay hosts, they will mutate to the acceleration speed of spreading among people. If we cut down their transmission pathway by a high-efficiency method such as lockdown strategy, they will mutate to exploit the already resided hosts, such as compete well inside the host with other in vivo mobile genetic elements and then become less transmissible. Unlike advanced infectious agents such as bacteria or fungi, viruses can only sustain on living beings, outside a living body they will lose their invading capability after a certain time. According to China's hand-on experience, both SARS in 2003 and SARS-VOR-2 today will finally wane or mutate to a less virulent strain after 6-12 months of a large-scaled lockdown to block the viruses get relay hosts. (This is somewhat similar to the "pneumonia calcification" process, just happens at a population level instead of individual human lungs with different species.)

From this fact we could understand, for any intervention method aim at COVID-19, such as vaccines, drugs, or medical devices must be mandatorily regulated to an over $99 \%$ block rate. This way is to block the fresh supply for the virus. It is highly risky to allow any vaccines or drugs that have failed to reach the "lockdown equivalent block efficiency" to be applied. Slip-through viruses with persistent fresh suppliers will mutate to make the vaccine, or the drug becomes ineffective. As is known, all antibiotic resistance, vaccine resistance, or drug resistance strains are unexceptionally born from this way. Such vaccine or drug-resistant mutations base on persistent fresh suppliers will finally make the removal of the infectious agent on the population level becomes extraordinarily challenging.

Presently popular vaccines intended for COVID-19, not only lower in placebo infection rate but also far inferior to the over $99 \%$ lockdown equivalent block efficiency, and their announced "effectiveness" is indeed not the "block rate", use of them will offer continuously fresh supply for sustaining the
COVID-19 strain and then accumulate mutations that counteract our interventions, and later become the next generation of pandemic infectious agents. Then all the people will be unintentionally busy in dealing with diverse COVID-19 mutations that impotent the vaccine every year, this is not a "decent job opportunity" for some people, but a devastating economic impact and future disaster to our human race. Vaccine methods are proper for some infectious diseases that can arouse lifetime immune protection, such as smallpox, the vaccine works out against it can reach a very high block rate. For problems that happen to a person many times in life such as the common flu, the immunization solution is truly challenging, and the final block rate will be accordingly lower. The claim that certain vaccines can reduce the infection rate by $60 \%$ is a kind of self-deception. Most people really don't know what such kind of claims means for the pandemic and what is immunosenescence. From a personal level, anyone who is vaccinated with a $90 \%$ block rate will still get the COVID-19 infection with a $10 \%$ chance. From the population level, a lower block rate vaccine will allow the viruses to get persistent fresh suppliers and then create the next generation of the pandemic agents in the future. If China adopted such a claim and waiting for this sort of lower block rate vaccines, it is impossible to see the largest population in the world have fully controlled the COVID-19 at the population level today! (China has never used any vaccines for COVID-19, at that time, no vaccine available; and while so many critically ill patients poured in to crash the capacity of all hospitals \& healthcare facilities, it is no chance for any vaccine to injection. Even a vaccine worked out at that time, it generally only fit for protecting healthy people, can't be injected into already infected \& symptom obvious SARS-VOR-2 patients, a vaccine will undoubtedly worsen the condition of these patients. The story of "herd immunity" is also terrible since this way has hiddenly accepted the "coexist" of the virulent agent with humans. We can deprive the sustaining sites of the RNA coronavirus by lockdown, why we pre-surrender the "coexist" state? And such a "coexist" still can't reduce immunecompromised or immunosenescence people percentage or "protect" them. In contrast, with a full lockdown strategy, even for Wuhan city that had once in a situation with over $40 \%$ infected rates at the population level in some regions, China has successfully waned the SARS -VOR-2 viruses by blocking their fresh supply at a population level. This strategy is so far the only successful one that has been validated at the population level in the world.)

As mentioned, a full lockdown is the only validated resolution for a pandemic; however, with a higher economic sacrifice that most nations can't afford, also quite unfeasible to execute for

\section{Volume 10 Issue 1, January 2021}




\section{International Journal of Science and Research (IJSR)}

ISSN: 2319-7064

SJIF (2019): 7.583

most countries. The three types of products which have been classified by Health Canada as Class II medical device, 319987HA\#, 319987HC\#, and 319987 UVD\#, are a set of "lockdown block efficiency equivalent devices" for COVID-19. (319987HA\#, adult use. 319987HC\#, children use.) They work together with proper handling can deal with COVID-19 or other equivalent infectious agents with an over $99 \%$ block efficiency, without considering social distancing.

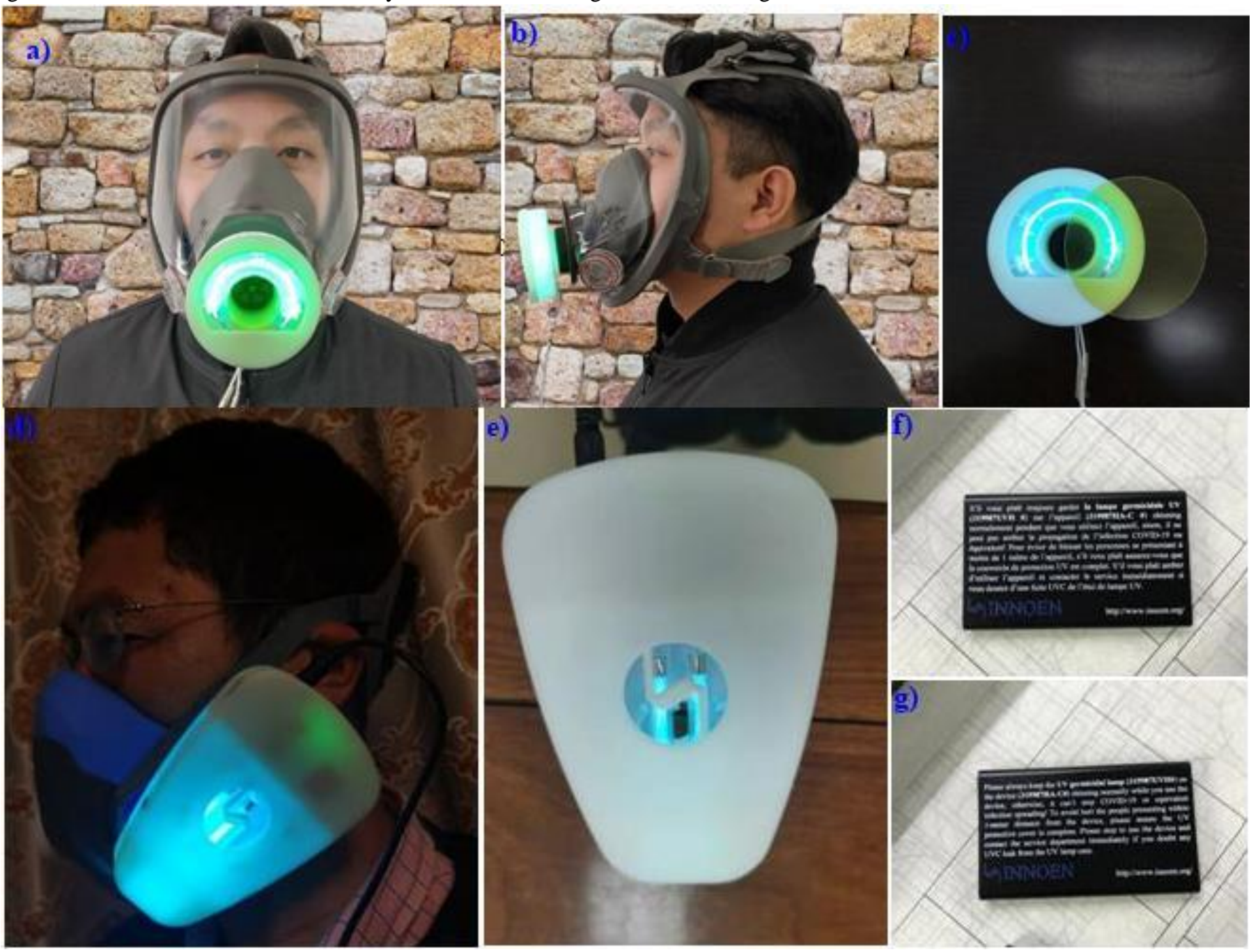

Figure 1: Innoen ${ }^{\circledR}$ COVID-19 airborne infection UV quarantine ${ }^{\circledR}$ devices (319987 HA\#) and the power bank

a) a 319987HA\# UV quarantine ${ }^{\circledR}$ device with one set of ring-shaped UVC germicidal lamp. b) side view of it. c) a ring-shaped UV germicidal lamp housing of the device with the UVC protective cover opened. d) a 319987HA\# UV quarantine ${ }^{\circledR}$ device with two sets of U-shaped UVC germicidal lamps. This type of design fits for those with glasses and also in a less infected region. However, while coronavirus concentration reaches a certain threshold, people must use devices with full eye covering like in Fig. a) \& b), the latter comply with the medical device definition from regulatory affairs. We should also note while facing bio-weapons' attack, this type of design must combine with eye goggles, human eyes are quite weak for aggressive infectious agents. e) one set of U-shaped UV germicidal lamp housing with the lamp shining inside it.f)-g) The products need a 10000mA power bank which can last for 8-12 hours. Each side of the power bank with the COVID-19 instruction on it.

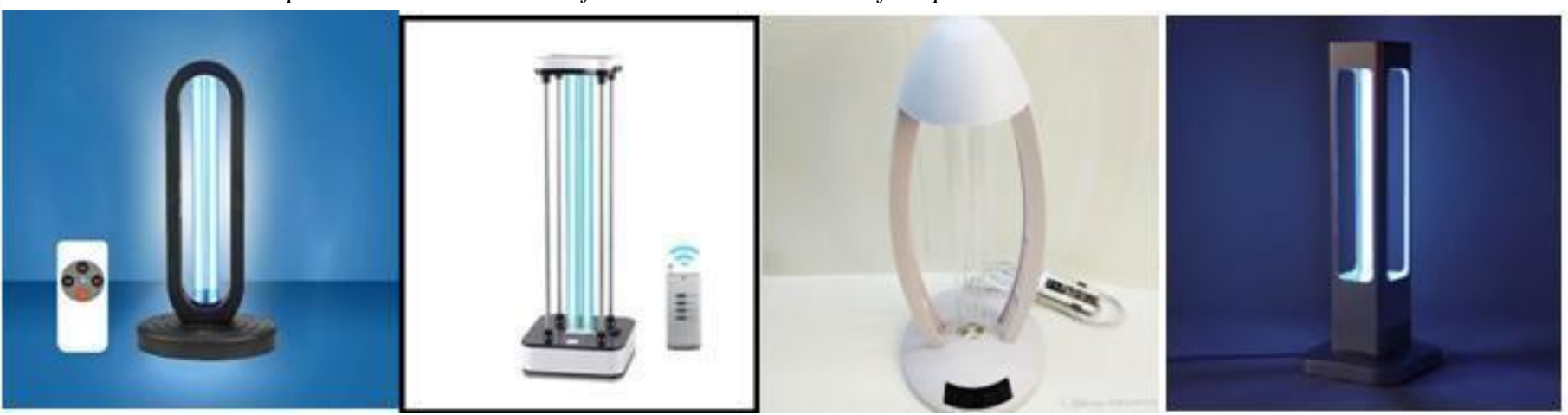

Figure 2 | Innoen ${ }^{\circledR}$ COVID-19 airborne infection UV quarantine ${ }^{\circledR}$ devices319987 UVD\#Desktop-type 319987UVD\# UV germicidal lamps with remote control are generally used for issuing 30min protocol for incoming outdoor clothes, goods, groceries, etc., each family can share one unit. They are compensation for airborne infection device 319987HA\# and 319987HC\# since they can deal with infectious agents beyond the airflow condition, very convenient for individual or family use. (The outside structures of the desktop UVC lamps are diverse, what critical is the 253.7nm UVC radiation strength. Be careful, not to allow the UVC radiation to shine on naked skin or eyes while using 319987UVD\#. They lack UV protective covers as those on 319987HA\#-HC\#, people either need to use a remote control or UV protection.)

\section{Volume 10 Issue 1, January 2021}

www.ijsr.net 
For 319987HA\#, 319987HC\#, each person per unit can use for over 12000 hours and the price is quite lower than all the medical-surgical masks with the same usage time, also more effective. And no matter how the infectious agents can mutate, every mutation including diverse artificially constructed bio-weapons will unexceptionally fail in front of these devices. (As is known, in a world with deep globalization, it is challenging to locate diverse bio-weapon attacks. The only solution is for everyone with a UV quarantine device, then no biological agent can invade and issuing an attack.) Furthermore, they can be easily used by the public as well as professionals with little training. (These devices have been using in China, Wuhan city since Oct.1, 2020; and in GTA, Ontario Canada on Jan. 15, 2021; will ship to all over the world later with regional regulatory approval.)

Please careful note, the primary purpose of 319987HA\#, $\mathrm{HC \#}$ is specifically designed for human nasal protection. People always live in the illusion that antibodies in the blood can protect the whole body from any infection. However, the fact is no matter how many human neutralizing antibodies (HuNAbs) inside the blood, either activated by vaccination, direct injection of HuNAbs, or accumulate from the last infection, they only can reduce infections in the lungs but can do nothing for the viruses in the nasal turbinates. The protection efficiency of blood HuNAbs for nasal and for lungs is totally different [1]. The nasal region becomes a harbor for the virus. For this reason, our device must be worn by both healthy and infected people without any test.

From here we can also understand the term "asymptomatic infection". While coronaviruses only reach and proliferate in the human nasal turbinates, the host will then issue "asymptomatic infection", undetectable even for a throat swab; however, still transmissible to others. Coronavirus will stay there for weeks or even longer depending on individual health conditions, only after the viruses further invade other parts of the body, a patient can be tested to confirm the COVID-19 infection. The nasal region becomes a temporary refuge for asymptomatic transmissible viruses. And it is still challenging to find out asymptomatic infection in an earlier nasal harbor stage by routine tests. Nucleic acid tests can't distinguish between dead or alive viruses. Possibly use the immunodeficiency animals to directly sample in the public regions, especially the human-animal interaction regions, is a solution for guiding UV quarantine devices to be used in an earlier stage to preclude a potential pandemic.

The fundamental reason why nasal turbinate protection can't get help from blood HuNAbs concentration is blood can't reach these regions. The fundamental reason why nasal turbinate protection can't get help from blood HuNAbs concentration is blood can't reach these regions. Inside human nostrils, there are a lot of nasal hairs which blood circulation can't access. (For animals, there are still exist outside vibrissae.) Therefore, no matter how many antibodies inside the blood, how powerful the antibodies to the virus, or how long these antibodies come from; the antibodies inside the blood can't reach the coronavirus on the nasal hairs and then can do nothing for these viruses.
On these dense and moisture nasal hairs, there are a lot of dander, detached epithelial cells, or secrets, etc., that benefit the survival of coronaviruses. These viruses can stay much longer than outside the human body. The SARS-VOR-2 viruses even possibly can proliferate on the nasal hairs with detached epithelial cells. The nasal hairs finally become the basecamp of viruses for later invading.

Some people have realized the weakness of the nasal region, then design nasal spraying vaccines. These types of products still do not solve the problem. Vaccines need to function with blood, on the nasal hair, no blood available, then these sprays can neither activate the immune reactions nor can kill the coronavirus on the nasal hair clusters. If people spray sanitizers instead of vaccines, then the users need to spray every 5 minutes while they expose to a high concentration of viruses. This way of using is unpracticable for protection.

The best way for nasal protection is still our UV quarantine devices. It can block airborne SARS-VOR-2 viruses in an extraordinarily higher efficiency before they can reach nasal hair. Even some viruses finally reach the nasal hair, the ozone produce by the UV quarantine devices persistently go through nasal hair will still kill them effectively.

Thanks for realizing the nasal protection function, pandemic block, and anti-bio-weapon roles of 319987HA\#, HC\#, also hope more people can work out more and more "Lockdown COVID-19 block efficiency equivalent" medical devices, vaccines, or drugs can be worked out!

Please remember, such a device or vaccine must demonstrate an over 99\% COVID-19 block rate at phase 3 clinical trial to qualify as the title "lockdown equivalent", never offering the viruses with any fresh supply!

\section{References.}

[1] Zhou, D.Y. et al. Robust SARS-CoV-2 infection in nasal turbinates after treatment with systemic neutralizing antibodies. Cell Host \& Microbe, 29, 1-13(2021). Doi:10.1016/j.chom.2021.02.019 